\title{
LISE MEITNER
}

\author{
LISE MEITNER
}

Maria Nigro

Universidad de Bari, Italia

\section{Riassunto:}

Lise Meitner, nacque nella Vienna imperiale nel 1878 da una famiglia ebraico-galiziana. $\mathrm{Fu}$ una delle prime donne a conseguire il diploma e ad entrare all'Università, dopo che venne abolita la legge che privava le donne dell'istruzione. Provava molta stima per Marie Curie, in quanto possedeva una grande intelligenza e conoscenza. La Meitner aveva un obbiettivo, sfidare il mondo maschile. Non ebbe un'esistenza facile e visse in una società non pronta ad accettare l'indipendenza femminile.

\section{Palabras claves:}

istruzione, mondo maschile, indipendenza femminile.

\section{Abstract:}

Lise Meitner was born in 1878 in Vienna, in a Jewish-Galician family. She was one of the first women who got the degree and who attended the University, after that the law, which deprived women of education, was lifted. She appreciated Marie Curie because of her intelligence and knowledge. Lise Meitner had a goal, challenging the male world. She did not have an easy life and she lived in a society which was not ready to accept female empowerment.

\section{KEY WORD:}

education, male world, female empowerment. 
Lise Meitner nasce il 7 novembre 1878 nella sfavillante Vienna imperiale. Nonostante i genitori, Philipp Meitner e Hedwig Skovran, siano entrambi di origine ebraico-galiziana è allevata in un clima di assoluta libertà religiosa. Lise vive una fanciullezza serena e mostra attitudine per la matematica già dai primi anni di scuola, ha una grande passione per la musica e una viva curiosità per i fenomen naturali. Secondo quanto riferisce un aneddoto, il gioco di colori creato da una macchia d'olio in una pozzanghera avrebbe acceso in lei la passione per la fisica (RIFE R., 1992, p. 21). La storia è poco credibile dato che, come risulta dai documenti, l'interesse iniziale di Meitner era rivolto alla matematica e che, solo in seconda istanza e per motivi pratici o fortuiti, abbia preso corpo il progetto di dedicarsi alla fisica. Nel 1892, ancora quattordicenne, nonostante gli eccellenti rendimenti scolastici, Lise è costretta a terminare gli studi. Il suo più grande desiderio, quello di conseguire la maturità scientifica, non è realizzabile. La legislazione dell'impero austro-ungarico non consente alle donne di frequentare né le normali scuole superiori, né l'università. S'iscrive, in alternativa, all'Akademischen Gymnasium, scuola viennese riservata alle ragazze, dove studia la lingua francese e nel 1896, all'età di diciotto anni, supera l'esame che le consente di insegnarla. Due anni dopo è finalmente varata la legge che abolisce il divieto per le donne austriache di frequentare liceo e università. Diverse ragazze seguono lezioni private per conseguire la maturità e avere accesso finalmente agli studi superiori. Lise è fra queste. Consegue la maturità l'undici luglio del 1901. Ha già 23 anni. Nell'autunno dello stesso anno s'iscrive all'Università (NIGRO M., 2002, p. 511).

La presenza di studentesse, in quell'anno di corso, lascia perplessi docenti e studenti. Lise e Selma Freud, le uniche donne iscritte a Fisica, si sentono addirittura delle "intruse" (KERNER C., 1999, p. 37). I problemi sono molteplici. La situazione è particolarmente difficile in una facoltà scientifica. Tanto per fare un esempio, i docenti non accordano facilmente fiducia a una donna; eppure, per partecipare attivamente alla ricerca, bisogna trovare un tutor. Molti studiosi sono convinti che le donne non abbiano le doti intellettuali per familiarizzare con la matematica e la pratica sperimentale Non è tutto. La necessità di frequentare i laboratori e di lavorare a contatto con elementi dell'altro sesso è vista come una complicazione, ulteriore e insuperabile. Le università austriache e tedesche sono state per secoli il tempio della cultura maschile. La legge, di fatto, non può abolire d'un tratto tutte le difficoltà. In realtà non bisogna dimenticare che, in quel tempo, la società non era ancora preparata, nemmeno a livello strutturale, per un'innovazione di questo tipo, per esempio la mancanza di

1 Uno spaccato del tempo lo offre una raccolta di circa cento interviste, realizzate da A. Kirchho nel 1897, allo scopo di saggiare l'opinione degli "intellettuali" sull'opportunità che una "donna" intraprenda la carriera scientifica: emerge la convinzione generalizzata della presunta "incapacità intellettiva" femminile, mentre risulta esaltato il ruolo della donna, moglie e madre (KIRSCHHOF A., 1897, pp. 256-257) servizi igienici per le donne, era un problema reale, che andava al di là di qualsiasi atteggiamento culturale preconcetto. Si tenga, comunque, ben presente che la possibilità concessa alle donne di accedere alla laurea non comportava la facoltà d'insegnare. La prima persona che ha tentato di dissuadere Lise dal proprio proposito è suo padre. L'avvocato Philip Meitner conosce molto bene la situazione politico-sociale dell' Austria e non le nasconde le difficoltà cui poteva andare incontro una giovane donna desiderosa di avviarsi a un percorso di tipo scientifico. Considera inoltre una seria aggravante l'esile struttura fisica della figlia. Avrebbe voluto che diventasse una pianista (SEXL L., HARDY A., 2002, pp. 7-20). Le sue richieste lo lasciano, pertanto, perplesso. Lise però dimostra d'essere tenace e di avere una vera e propria passione per la scienza. Lise Meitner è innegabilmente una donna che ha vissuto fuori degli schemi sociali tipici del suo tempo. Ha scelto di non sposarsi e di non avere figli anche se la realizzazione di un tale proposito l'ha inevitabilmente costretta a superare numerose difficoltà. Il progetto esistenziale di Lise Meitner, per l'epoca in cui la nostra protagonista ha vissuto, è oggettivamente "audace". Lise vuole confrontarsi con gli uomini su di un terreno notoriamente ostico. Non è semplice. Negli ambienti colti della Vienna del secolo XIX cresce quotidianamente l'interesse per la tecnica e per la scienza, vi sono però ancora molti pregiudizi di fronte a donne propense a fare scelte tradizionalmente riservate agli uomini. Meitner, suo malgrado, è dunque protagonista di un meccanismo che tenta d'imprimere un'accelerazione a un cambiamento sociale e culturale già in atto ma in ogni modo difficoltoso e complesso

È molto probabile che la giovane Lise sia rimasta affascinata dalla figura di Mania Sklodowska Curie. I giornali di tutta Europa, tra la fine del secolo XIX e l'inizio del $X X$, narrano con enfasi la straordinaria avventura scientifica di questa donna fuori dal comune. Anche se è assolutamente fuori discussione un confronto fra queste due grand figure di donna: Madame Curie quando lascia Varsavia per Parigi opera una scelta politica. La Francia era il paese dell'illuminismo, della rivoluzione, del positivismo sociologico e soprattutto dei diritti civili garantiti a tutti. Tutti questi motivi hanno spinto alle proprie scelte l'esule polacca. Il "caso" ha fatto il resto. L'incontro della vivace Mania Sklodowska con Pierre Curie e l'inaspettato quanto brillante risultato conseguito da Henri Becquerel nel 1896, costituiscono le due circostanze che hanno conferito una svolta particolarmente favorevole alle scelte e alla carriera di Madame Curie. Per Lise Meitner le cose sono andate in tutt'altro modo. Sin da ragazza era consapevole dei suoi interessi e delle sue passioni. Vuole fare lo scienziato. Diventare allieva dell'illustre fisico austriaco Ludwig Boltzmann è pertanto una scelta consequenziale. Una serie di circostanze l'ha, poi, persuasa a convertirsi all'atomismo quando parlare d'atomi, in sede di scienza fisica, era ancora quasi un sacrilegio. Questa sua posizione teorica è probabilmente fra i motivi, ma non sicuramente il solo, che hanno indotto, in un primo momento, lo stesso Max Planck a non voler accettare Meitner come propria allieva. 
Le vicissitudini di Lise Meitner, a prima vista, potrebbero sembrare piuttosto simili a quelle di molte altre donne che hanno lottato per l'affermazione dei propri diritti. Difficoltà sociali e culturali particolarmente pregnanti hanno segnato la sua lunga esistenza che presenta, invece, tratti d'autentica singolarità. Senza voler nulla togliere al carattere fiero e combattivo del personaggio, al suo impegno, sacrificio e vivacità d'ingegno, se differenza esiste fra le vicende umane di quest'ultima e quelle delle altre donne, questa differenza consiste nel fatto che Lise Meitner è stata in buona sostanza una privilegiata. Lise è figlia di un professionista noto, stimato, benestante e soprattutto di mentalità aperta. Tutto ciò si è tradotto in una lunga serie di vantaggi dei quali non è possibile calcolarne l'effettiva portata. Tra questi è giusto annoverare la possibilità di studiare "privatamente" e di essere sostenuta economicamente dalla famiglia anche nel progetto di recarsi a studiare all'estero. Dopo il conseguimento della laurea, sollecitata dal grande chimico austriaco Stefan Meyer, in seguito alla prematura morte di Boltzmann, ha il privilegio d'essere "pioniera" dell'atomismo in fisica. A Berlino, dove si reca con l'intento di studiare con Max Planck, del quale diverrà assistente, la prima donna in Prussia, incontra Otto Hahn, apprezzato allievo di Ernest Rutherford e promettente chimico nucleare.

I privilegi di partenza hanno dunque consentito a Meitner di essere guidata dal fior fiore dell'aristocrazia intellettuale della scienza europea del primo Novecento. Non è certamente poco. Considerazioni analoghe possono, a mio giudizio, farsi a proposito della politica antiebraica della Germania nazista. Non vi è alcun dubbio che vedersi togliere titoli scientifici e accademici faticosamente conquistati, essere costretti all'esilio attraverso la fuga clandestina siano elementi che segnano la vita di qualsiasi individuo. Ancora una volta però è giusto rilevare quanto la Meitner sia stata privilegiata. Grazie a Planck, von Laue e Otto Hahn, rimane presso il Kaiser Wilhelm Institut für Chemie per ben sei anni di regime nazista. Non si dimentichi poi che proprio questa circostanza le ha permesso, tornando a lavorare con Hahn, di partecipare attivamente alle ricerche sulla radioattività artificiale e sugli elementi transuranici che troveranno il loro epilogo nella scoperta della fissione del nucleo atomico. Paradossalmente quest'ultimo evento ha giocato un ruolo tutto particolare a danno della scienziata. Meitner è stata la prima a comprendere il meccanismo teorico della fenomenologia della fissione nucleare. Questo merito le è stato riconosciuto con notevole e imperdonabile ritardo. C'è di più. Pur non avendo partecipato al "Progetto Manhattan", è stata poi additata come "la madre della bomba atomica" (SEXL L., HARDY A., 2002, pp. 7-20).

$E^{\prime}$ anche questo un problema che merita qualche ulteriore ragguaglio e un più sereno giudizioinsedediStoriadellaScienza.Laletteraturastatunitense, inprimis, harecriminato a gran voce la sottrazione del Premio Nobel a Lise Meitner per la spiegazione teorica della fissione nucleare. La suddetta questione è stata ripresa con una certa veemenza in tempi recenti, probabilmente alimentata da un certo tipo di "esigenze editoriali" che privilegiano l'aspetto sensazionale rispetto a un pacato e approfondito approccio storico. Non trascurabile in questo fenomeno è lo sviluppo delle cosiddette "ricerche di genere" che hanno preso piede inizialmente in America e, poi, in tutta Europa sulla spinta del movimento femminista. In seguito a quest'onda emotiva emerge in tutta la sua drammaticità la figura di Lise Meitner, le cui vicissitudini offrono tutti presupposti per fungere da modello al nuovo fenomeno della "donna vittima a tutti i costi" di impedimenti culturali e sociali in un ostile mondo scientifico gestito dagli uomini. Nel 1977 su Physics Today esce un articolo che non lascia alcun dubbio riguardo la tesi che gli autori intendono sostenere: Una storia Nobel d'ingiustizia del dopo guerra (CRAWFORD E., SIME R.L., WALKER M., 1997, pp. 26-32). I documenti ufficiali delle discussioni della Reale Accademia Svedese delle Scienze, per l'assegnazione del Premio Nobel, sono normalmente tenuti riservati per cinquant'anni, solo dopo questo lasso di tempo sono messi a disposizione degli interessati. Quelli riguardanti il 1945 e il 1946, pertanto, sono stati resi accessibili negli anni in cui il suddetto lavoro veniva pubblicato. Gli autori, Ruth Lewin Sime, Elisabeth Crawford e Mark Walzer scrivono che Meitner nonostante nell'estate del 1938, quando si aggrava la posizione degli ebrei in Germania, sia costretta a lasciare Berlino per rifugiarsi a Stoccolma, non interrompe la collaborazione con il Kaiser Wilhelm Institut für Chemie. Lo testimonierebbe la corrispondenza, particolarmente intensa in quel periodo, con Otto Hahn. La scoperta della fissione del nucleo viene pubblicata però solo a nome di Hahn e Strassmann. Meitner e Frisch, immediatamente dopo, forniscono la prima interpretazione teorica del processo di fissione.

Secondo la ricostruzione di Sime, Crawford e Walker tra il 1940 e il 1943, quando numerose proposte internazionali suggeriscono che Meitner venga ricompensata con un premio Nobel, il comitato per la fisica obietta che la scoperta riguarda solo la chimica. Sostanzialmente l'argomentazione è che il lavoro di Hahn è di grande interesse, mentre il lavoro di Meitner e Frisch non è straordinario e se c'è un contributo teorico significativo, è quello reso, in seguito, da Bohr che, riferendosi all'articolo di Meitner e Frisch, aveva indicato l'ingegnosa spiegazione come il punto di partenza per la futura teoria nucleare. I due commissari, in tal modo, commentano Sime e colleghi, avrebbero distorto e sminuito i contributi di Meitner e Frisch. La disgiunzione dei contributi di Hahn e Strassmann da un lato, e Meitner e Frisch dall'altro, avrebbe diviso, secondo gli autori del suddetto articolo, la scoperta della fissione nucleare tra chimica e fisica, tra esperimento e teoria, tra tedeschi ed esuli.

Lo storico Robert Marc Friedmann ci offre un più convincente punto di vista. Secondo Friedmann la Svezia, come anche molti altri Stati, immediatamente dopo la seconda guerra mondiale aumenta notevolmente gli investimenti per la ricerca a sostegno della 
difesa e dell'autonomia del paese. Il grande ottimismo che si diffonde sulle possibili applicazioni dell'energia nucleare, incita a realizzare grandi e costose installazioni per lo studiodell'atomo.Gliscienziatinuclearipiù autorevoliinstauranofortilegamitra scienza, industria e milizia. La nomination Nobel di Meitner avrebbe prodotto seri problemi. Dal sei agosto 1945, giorno dello scoppio della bomba atomica, Lise si prodiga contro la corsa agli armamenti nucleari, suggerendo una Commissione Internazionale per il Controllo Nucleare (CHIU S.C., 1994, p. 102). In un'Europa protesa verso il nucleare Meitner era un personaggio scomodo. Un premio di tale rilevanza avrebbe trasformato la misera rifugiata in un'autorità internazionale nel settore della fisica nucleare. Questa prospettiva avrebbe messo in discussione molti progetti. Motivi politici, dunque, secondo gli studi di Friedmann, avrebbero tolto a Meitner ogni possibilità di ottenere l'ambito riconoscimento per i propri contributi scientifici. Lise ricevette, comunque, numerosi premi prestigiosi sia in Svezia che all'estero. Le fu, inoltre, assegnata una piccola unità di ricerca nel laboratorio di scienze dell'ingegneria presso la Reale Accademia svedese. Morirà, nel 1968, alla veneranda età di novant'anni a Cambridge dove si era ritirata per stare vicina al nipote Otto Robert Frisch (FRIEDMANN R.M., 2005, pp. 41-60). La travagliata e intensa esistenza di questo "fragile gigante" è stata sì segnata dall'accanimento semitico e da interessi politici ma resta, indelebilmente, scolpita nella Storia della Scienza.

\section{RIFERIMENTI BIBLIOGRAFICI}

Kirschoff, A., Die Akademische Frau, Berlin, Hugo Steinik Verlag, 1897.

Rife, P., Lise Meitner. Ein Leben für die Wissenschaft, Hildesheim, Claassen, 1992.

Chiu, S., Lise Meitner. Die Trümmer meiner Vergangenheit, in Frauen im Schatten, Wien, Dachs Verlag GmbH., 1994.

Crawford, E., Sime, R.L., Walker, M., "A Nobel Tale of Postwar Injustice", in Physics Today, CCCLXXXII (1997), pp. 26-32.

Friedman, R.M., The Politics of Excellence: Behind the Nobel Prize in Science, New York, Henry Hotl, 2001.

----, Ricordando Lise Meitner, a cura di P. Govoni e G. Pancaldi, Pendragon, Bologna, 2005.

Kerner, C., Lise, Atomphysikerin, Basel, Beltz Verlag, 1999.

Nigro, M., "Lise Meitner. Donna, ebrea, scienziato", in Physis, XXXIX (2002), pp. 511528.

Sexl, L., Hardy, A., Lise Meitner, Reinbeck bei Hamburg, Rowohlt Verlag, 2002.

Sime, R. L., Lise Meitner. Ein Leben für die Physik, Frankfurt am Mein-Leipzig, Insel Verlag, 2001. 